See discussions, stats, and author profiles for this publication at: https://www.researchgate.net/publication/8074204

\title{
Predicting Age-Related Dual-Task Effects With Individual Differences on Neuropsychological Tests.
}

Article in Neuropsychology · February 2005

DOI: 10.1037/0894-4105.19.1.18 - Source: PubMed

\section{CITATIONS}

67

3 authors:

Roee Holtzer

Yeshiva University

134 PUBLICATIONS 7,385 CITATIONS

SEE PROFILE

Brian C Rakitin

Columbia University

51 PUBLICATIONS 2,587 CITATIONS

SEE PROFILE

Some of the authors of this publication are also working on these related projects:

Project Cognitive Reserve View project

Project Cognitive remediation to improve simple and complex locomotion View project
Yaakov Stern

Columbia University Vagalos College of Physicians and Surgeons 1,023 PUBLICATIONS 83,244 CITATIONS

SEE PROFILE 


\title{
Predicting Age-Related Dual-Task Effects With Individual Differences on Neuropsychological Tests
}

\author{
Roee Holtzer \\ Columbia University College of Physicians and Surgeons \\ and Yeshiva University
}

\author{
Yaakov Stern and Brian C. Rakitin \\ Columbia University College of Physicians and Surgeons
}

\begin{abstract}
This study examined the relation of dual-task performance to individual differences on neuropsychological tests. Neuropsychological test scores from 16 young and 16 older participants were simultaneously submitted to a factor analysis that yielded 2 factors (Attention/Executive and Memory) that differed by age and 2 (Motor Speed and Cognitive Status) that did not. Regression analyses revealed that these factors were significant predictors of performance on a delayed visual recognition task, but the relationship varied as a function of task condition. The Memory and Motor Speed factors were the strongest predictors of single-task performance, but the Attention/Executive factor was the most important predictor of dual-task performance. The authors conclude that compromised central executive may underlie age-related decline in dual-task performance.
\end{abstract}

Many studies have documented increased dual-task costs in aging (for recent meta-analyses, see Verhaeghen \& Cerella, 2002; Verhaeghen, Steitz, Sliwinski, \& Cerella, 2003), but discrepant views have been offered to account for this robust aging effect. Although dual-task methodology has been used to evaluate defined facets of the executive functions (e.g., Baddeley, 2002; Baddeley \& Hitch, 1974; Meyer \& Kieras, 1997; Miyake et al., 2000), the relation of dual-task performance to neuropsychological measures, in general and with respect to aging in particular, has not been extensively studied. This is surprising given the interest in the executive function (Salthouse, Atkinson, \& Berish, 2003; Stuss \& Alexander, 2000; Stuss \& Levine, 2002) and the possibility that an informative relation between standardized neuropsychological tests and dual-task performance may exist.

In the present article, we evaluated whether individual differences in neuropsychological tests in young and older individuals predict and characterize age-related differences in chronometric and discrimination performance indices in a dual-task paradigm. We begin by reviewing present accounts of dual-task performance decrements in aging and previous studies that evaluated the relationship between dual-task performance and neuropsychological tests.

Roee Holtzer, Cognitive Neuroscience Division of the Taub Institute, Columbia University College of Physicians and Surgeons, and Ferkauf Graduate School of Psychology and Department of Neurology, Albert Einstein College of Medicine, Yeshiva University; Yaakov Stern, Cognitive Neuroscience Division of the Taub Institute and Departments of Neurology and Psychiatry, Columbia University College of Physicians and Surgeons; Brian C. Rakitin, Cognitive Neuroscience Division of the Taub Institute and Department of Neurology, Columbia University College of Physicians and Surgeons.

This work was supported in part by Federal Grant T32 AG00261 to Yaakov Stern.

Correspondence concerning this article should be addressed to Brian C. Rakitin, 630 West 168th Street, P \& S Box 16, New York, NY 10032. E-mail: br130@columbia.edu

\section{Age-Related Decline in Dual-Task Performance}

Age differences in dual-task performance have been studied extensively (for a review, see Hartley, 1992; McDwod \& Shaw, 2000). Whereas some researchers have failed to demonstrate agerelated decline in dual-task performance (e.g., Nyberg, Nilsson, Olofsson, \& Backman, 1997; Somberg \& Salthouse, 1982; Tun \& Wingfield, 1994; Wickens, Braune, \& Stokes, 1987), many have found that aging is associated with increased dual-task costs (e.g., Crossley \& Hiscock, 1992; Li, Lindenberger, Freund, \& Baltes, 2001; Salthouse, Rogan, \& Prill, 1984; Whiting \& Smith, 1997). Hartley (1992) suggested several reasons why age-related costs might be noted in dual-task paradigms even though these costs are not attributable to divided attention. These include compromised performance by elderly adults on the individual tasks used in the paradigm, variable complexity levels of the individual tasks, and use of two tasks that target the same perceptual modality. Hartley and Little (1999) found that age differences in dual-task costs were reduced significantly when using two relatively simple tasks that are presumably processed via different perceptual modalities. Hartley (2001) suggested that age differences in dual-task costs may be confined to concomitant generation and execution of two similar motor programs. This suggestion, however, can be directly evaluated in studies in which each individual task is processed via different stimulus response channels.

Although several mechanisms have been offered to explain age-related differences in dual-task performance, they are not without criticism. Salthouse $(1985,1996)$ argued that age-related dual-task costs reflect a general reduction in speed of cognitive processing associated with age. However, Kramer, Hahn, and Gopher (1999) demonstrated that age-related performance differences in a task-switching paradigm could not be accounted for by general slowing. Instead, they suggested that executive control in aging appeared compromised when memory load increased.

The complexity hypothesis (McDwod \& Craik, 1988; McDwod, Vercruyssen, \& Birren, 1991) suggests that dual-task costs can be expected to increase as a function of task complexity. However, Morris, Gick, and Craik (1988) and Gick, Craik, and Morris (1988) found that increased complexity and dual-task interference were 
differentially related to aging. In those two studies, increased syntactic complexity but not dual-task interference resulted in age-related performance decrements. Navon and Miller (1987) suggested that dual-task costs in old age result from a limited capacity to organize and execute motor responses. Lack of flexibility in switching attentional resources between tasks (Kortling, 1991 ) and the proclivity for a conservative response bias (Batsakes \& Fisk, 2000; Glass et al., 2000) were also offered as possible contributors to age-related decline in dual-task performance.

Two recent studies showed that age differences in dual-task costs, as measured by central tendency (Anderson, Craik, \& Naveh-Benjamin, 1998) and dispersion parameters (Anderson, 1999) of reaction time, varied depending on whether the interference occurred during encoding (i.e., stimulus presentation), recall, or recognition (i.e., probe presentation) components of a working memory task.

The above short review of possible reasons for the effect of aging on dual-task performance illustrates the lack of consensus in the literature concerning this issue. Some of this inconsistency may be attributed to variability in dual-task paradigms, extent of training, and characteristics of the single tasks. Nonetheless, pursuing further explanations for this aging effect that may generalize beyond task-specific characteristics and variability in experimental designs is of great value. To achieve this goal, in the present study we took a different approach to further our understanding of why dual-task performance is reduced because of the aging process. We asked whether individual differences on empirically derived neuropsychological factors that represented different cognitive domains predicted or explained age-related differences in dual-task performance costs.

\section{Relations Between Dual-Task Measures and Neuropsychological Tests}

Dual-task or time-sharing ability has been traditionally thought to represent limited capacity and attentional resources. Moreover, theoretical models using specific dual-task paradigms as proxies of executive control (e.g., Baddeley, 2002; Baddeley \& Hitch, 1974) conceptualize this higher order cognitive ability in terms of a supervisory activating system (see Norman \& Shallice, 1980), which allocates attention to competing task demands. Hence, above and beyond task-specific characteristics, a relation between neuropsychological tests of attention and executive functions and dual-task measures may be expected.

However, we were able to find only a few studies that reported on the relation between performance on neuropsychological tests and dual-task measures. McDowell, Whyte, and D'Esposito (1997) found correlations between dual-task measures and neuropsychological tests of executive functions in patients with traumatic brain injury but not in healthy control participants. Bull and Scerif (2001) reported that with the exception of a small correlation with one performance index of the Stroop task, dual-task performance did not correlate with a number of neuropsychological tests or with mathematics ability in school children. Miyake et al. (2000) used a confirmatory factor analytic technique to derive three latent variables (inhibition, updating, and shifting) that were used to predict performance on a number of executive functions tests, including dual tasks in a college population. They found that the three latent variables contributed differentially to performance on complex executive tasks but were unrelated to dual-task performance. Malapani, Pillon, Dubois, and Agid (1994) reported that dual-task but not single-task reaction time measures correlated with Wisconsin Card Sorting Test performance indices in patients with Parkinson's disease and in control participants. Finally, Tun, O'Kane, and Wingfield (2002) found that performance of young and older participants on the Trail Making Test (Trails; Lezak, 1995) was related to recall accuracy when study conditions included meaningful distractors but not when the to-be-remembered information was presented in a quiet setting without interference.

The paucity and inconclusiveness of research examining the relation of neuropsychological tests to dual-task measures preclude any conclusions with respect to this topic. However, if an informative association can be demonstrated in samples of young and older individuals, it might shed light on factors that are relevant to dual-task processing in general and to age-related decline in dualtask performance in particular.

\section{Present Study}

The present study examined whether individual differences on empirically derived neuropsychological factors predicted age-related differences in chronometric and discrimination performance indices in a dual-task paradigm. Neuropsychological test scores from young and older individuals were included in the same factor analysis. The resultant factors represented individual differences in neuropsychological tests that were both related and not related to age. The relation of dual-task performance to individual expression of the factors was assessed in the context of whether age differences on those factors were significant. The factor scores were used to predict performance in the single- and dual-task conditions of a delayed visual recognition task (DVRT). This approach addressed the issue of whether the relation of individual neuropsychological factors to performance on the DVRT varied as a function of task condition.

We were interested in addressing the following questions and hypotheses:

1. Do individual differences on empirically derived neuropsychological factors predict dual-task performance of young and older participants? Or stated differently, can individual differences on those factors serve as adequate proxy for the effect of age on dual-task performance? We hypothesized that because some neuropsychological tests are sensitive to the effect of aging, performance on those tests would predict age-related differences in dual-task performance.

2. What neuropsychological factors were differentially related to DVRT performance in the single- and dual-task conditions? We hypothesized that because different demands were presumed to underlie single- and dual-task conditions, the relation of individual neuropsychological factors to DVRT performance would vary as a function of task condition. Specifically, in light of the presumed role of attention in allocating resources to competing tasks in dual-task paradigms, we hypothesized that the role of attention in predicting performance would be most potent in the dual-task condition. In contrast, we predicted that the Memory factor would be the best predictor of performance in the single-task condition given that the DVRT, when performed alone, is predominantly a memory test. 
3. Does aging have an effect on dual-task performance that is independent of the variance captured by the neuropsychological factors? We hypothesized that an age variable that was orthogonalized with respect to the neuropsychological factors would have an additional independent contribution to predicting dual-task performance. We expected that other factors, such as endurance or arousal, which are not typically captured by neuropsychological factors measures, would contribute to the effect of aging on dualtask performance.

\section{Method}

\section{Participants}

Sixteen older adults, ages $65-85$ years, and 16 younger adults, ages 19-31 years, participated in the present study. The older participants were community residents who were recruited from newspaper advertising and senior centers and who had previously participated in studies conducted at the Columbia Presbyterian Medical Center, New York. The young participants were undergraduate students at Columbia University.

All the participants were determined to be in good health on the basis of self-report and clinical interviews. Demographic information and summary statistics of the neuropsychological tests, per age group, are presented in Table 1.

Table 1 reveals that the young and older participants were comparable in terms of their education, estimated Verbal IQ (the National Adult Reading Test [NART]; Nelson, 1982), current cognitive status (the Dementia Rating Scale [DRS]; Mattis, 1988), and gender distribution within each group. Exclusionary criteria were medical and psychological history that might affect cognition (e.g., brain trauma, neurodegenerative diseases, and depression), medications known to have an effect on test performance, and

Table 1

Descriptive Statistics of Demographic Variables and Neuropsychological Tests (Using Raw Scores) per Age Group

\begin{tabular}{|c|c|c|c|c|}
\hline \multirow[b]{2}{*}{ Variable } & \multicolumn{2}{|c|}{ Young } & \multicolumn{2}{|c|}{ Older } \\
\hline & $M$ & $S E$ & $M$ & $S E$ \\
\hline Age** (years) & 21.2 & 0.76 & 74.3 & 1.40 \\
\hline Education (years) & 14.8 & 0.36 & 15.9 & 0.39 \\
\hline$\%$ female & 62.5 & & 62.5 & \\
\hline DRS total score & 142.12 & 0.37 & 140.56 & 0.93 \\
\hline NART total score & 39.25 & 1.07 & 39.87 & 1.65 \\
\hline BNT total score & 15.00 & 0.00 & 14.81 & 0.13 \\
\hline Digit Span $(\mathrm{FW})^{* *}$ & 13.12 & 0.59 & 10.87 & 0.49 \\
\hline Digit Span $(\mathrm{BW})^{* *}$ & 9.18 & 0.52 & 7.18 & 0.44 \\
\hline Trails (Form A) time $(\mathrm{s})^{* *}$ & 20.81 & 1.26 & 41.87 & 2.55 \\
\hline Trails (Form B) time $(\mathrm{s})^{* *}$ & 41.81 & 2.62 & 99.86 & 11.03 \\
\hline Trails $(\mathrm{B}-\mathrm{A})$ Time $(\mathrm{s})^{* *}$ & 21.00 & 1.91 & 58.46 & 9.73 \\
\hline SRT total recall** & 58.31 & 2.52 & 44.75 & 1.90 \\
\hline SRT saving score* & 0.92 & 0.02 & 0.73 & 0.06 \\
\hline Rey copy total score** & 35.06 & 0.33 & 32.62 & 0.61 \\
\hline Rey 30 -min delay total score** & 25.68 & 1.30 & 11.09 & 1.51 \\
\hline Reaction time*: $M(\mathrm{~ms})$ & 321.09 & 6.03 & 408.99 & 29.78 \\
\hline Reaction time: $S D(\mathrm{~ms})$ & 55.74 & 4.90 & 101.09 & 24.69 \\
\hline
\end{tabular}

Note. Independent-samples $t$ tests $(d f=30)$ were used to examine age differences on all measures with the exception of gender ratio. DRS = Dementia Rating Scale; NART $=$ National Adult Reading Test; BNT $=$ Boston Naming Test; FW = Forward; BW = Backward; Trails = Trail Making Test; SRT $=$ Selective Reminding Test.

$* p<.05 . \quad * * p<.01$. history of learning disability. All participants provided informed consent, and all were compensated for their participation.

\section{Neuropsychological Measures}

The neuropsychological test battery was selected to assess premorbid and current level of cognitive status and the following domains: attention, memory, language, executive function, and reaction time. Several issues were considered when selecting specific tests. First, tests were selected to cover neuropsychological domains that were thought to be related to the single- and dual-task conditions of the DVRT. It was important to select tests that were and were not sensitive to aging so that individual differences (as captured by the factor scores; see the Statistical Analyses section for details) would be both related and unrelated to age. When possible, we selected tests that have been used in our center in numerous prospective and cross-sectional studies of aging to ensure their adequacy for the study population and consistency with other ongoing research. Although the neuropsychological battery is not exhaustive, it provided sufficient information about the participants' overall neuropsychological profile.

The following tests were included in the neuropsychological battery. The NART (Nelson, 1982) was administered to estimate verbal intellectual functioning. The dependent measure was the total number of words read correctly. The DRS (Mattis, 1988) was used as a screen for current level of cognitive status. The total DRS score served as the dependent measure. The Digit Span Test (Wechsler Adult Intelligence Scale-Third Edition [WAIS-III]; Wechsler, 1997) total raw scores for the Forward and Backward conditions assessed span of verbal recall. A 15-item abbreviated version of the Boston Naming Test (Stern et al., 1992) was used to evaluate word-finding ability. The Selective Reminding Test (SRT; Bushke \& Fuld, 1974) assessed verbal memory. Total recall score and a saving score (number of items recalled after a delay and number of items recalled in the last trial of the immediate recall condition) served as dependent measures. The Rey Complex Figure (Rey, 1941), copy version (total score), was used to estimate construction and executive function. The Rey 30-min delay (total score) was used to evaluate delayed visual memory. Trails (Forms A and B; see Lezak, 1995) measured attention and executive function. The time required to complete each form served as the dependent measure. The time required to complete Form $\mathrm{A}$ was subtracted from the time required to complete Form B to provide a better measure of set-shifting ability. A computerized simple reaction time test (see Doran, Van Dongen, \& Dinges, 2000) assessed speed of processing. This simple vigilance reaction time task ran for $6 \mathrm{~min}$. Trials occurred with an intertrial interval of 2-10 s. The stimulus consisted of a large red $X$ that appeared in the center of the screen. Participants were instructed to press the space bar as quickly as possible when the stimulus appeared. After each response, the program provided response time feedback in milliseconds. Participants were instructed to respond as quickly as possible.

\section{Cognitive Single- and Dual-Task Measures}

The individual tasks used in the dual-task paradigm were designed to be consistent with Baddeley and Hitch's (1974) conceptualization of the central executive (for details regarding the theoretical basis and procedures of the cognitive tasks, see Holtzer, Stern, \& Rakitin, in press). That is, one task was presumed to represent the visuospatial sketch pad, and the other represented the phonological loop. The function of the central executive is to allocate and coordinate resources to the concomitant processing of two tasks, which presumably represent separate perceptual modalities. Moreover, designing the dual-task paradigm in this manner was intended to ensure that dual-task costs would represent the function of executive processes and not limited capacity of one modality.

\section{Apparatus}

A Macintosh iBook computer, with a 12.1-in. (31.3-cm) diagonal viewable monitor, was used to administer the single and dual tasks. A delayed 
visual recognition procedure and an auditory digit recall task were implemented using PsyScope software (Cohen, MacWhinney, Flatt, \& Provost, 1993). Visual stimuli were presented on the computer screen. Keys on the left and right sides of the keyboard served as response keys for the visual task. Auditory stimuli for the Digit Span Test were digitized voice recordings played by the computer and amplified by digital speakers.

\section{Task Procedures}

All training and testing procedures were conducted in the same laboratory room in the medical center. Each participant was tested individually. The training and testing of the single-task condition of the DVRT were completed in the first experimental session. The training and testing procedures of the DVRT in the dual-task condition were administered in the second experimental session. The single- and dual-task conditions of the DVRT are presented schematically in Figure 1.

The single-task condition of the DVRT (see Figure 1A) is a nonverbal adaptation of the Sternberg task (Sternberg, 1966, 1969). Each trial consisted of an encoding, retention, and probe phase. In the encoding phase, two or four visual stimuli were presented. The probe item either matched (true positive) or did not match (true negative) one of the stimuli from the study set. The participants indicated whether the probe item was included in the initial set by a differential button press. The participants were instructed to respond as quickly as possible.

The DVRT consisted of 60 trials and was conducted twice as a single task. The first run was designated as the training procedure, and the second run served as a baseline comparison to the dual tasks. Twelve Microsoft Word symbol characters served as stimuli for the DVRT. These symbols were thought to be nonverbal to the extent that they did not correspond to or intuitively relate to real words.

A computerized digit span served as the interference task. It consisted of auditory presentation and verbal recall of five-digit sets. The participants listened to a set of five numbers, produced by the computer at a rate of one digit per second. They were then asked to repeat the digits in the same order and at the same pace. The times at which each digit was produced was monitored by the computer. The accuracy of the digit recall was hand-recorded by the tester.

As with the DVRT, the computerized digit span task was conducted alone twice, with training preceding the testing condition. Each task consisted of 25 different strings that were five digits long. Randomization within each digit set was constrained by two factors: digits presented one after the other could not be consecutive either in descending or ascending order, and identical pairs of digits were not presented in consecutive digit sets.

With no exception, performance on the computerized digit span task when administered alone was at $95 \%$ accuracy or higher. Accuracy was reduced in the dual-task condition, especially for the older participants, but not below $85 \%$.

The dual-task condition of the DVRT consisted of 60 trials as well. It is presented schematically in Figure 1B. Participants were required to encode the visual study set while listening to the first set of digits, recall the digits during the first $5 \mathrm{~s}$ of the retention period, attend to a second set of digits during the last $5 \mathrm{~s}$ of the retention interval, and then recall the digits while making a decision about the identity of the probe.

\section{Experimental Procedures}

The first and second experimental sessions for each participant were conducted at the same time of day. Once written consent was obtained at the beginning of the first session, the tester conducted a comprehensive interview to obtain demographic, medical, and psychological information to ensure compliance with the inclusion and exclusion criteria of the study. Following the interview, the training and testing procedures of the DVRT in the single-task condition were administered. Subsequently, the participants completed the neuropsychological testing battery. The training and testing procedures of the DVRT in the dual-task condition were administered on the second day of testing.

\section{Statistical Analyses}

Chronometric and discrimination indices in the single- and dual-task conditions of the DVRT. First, it was necessary to demonstrate that performance differences between the young and older participants on the single- and dual-task conditions of the DVRT were statistically significant. DVRT reaction time data for each participant was filtered so that responses that were two standard deviations above an individual cell mean $(95 \%$ confidence interval) were eliminated. Responses that were under $150 \mathrm{~ms}$ were eliminated as well. Natural $\log$ transformation $\left(\log _{e}\right)$ of raw reaction time data was used to minimize group differences in the dual-task condition that were proportionate or disproportionate to group differences in the single-task condition (see Baddeley, Baddeley, Bucks, \& Wilcock, 2001).

Signal-detection theory assuming logistic distribution was used to calculate a discrimination index with the accuracy data of the DVRT in the

A

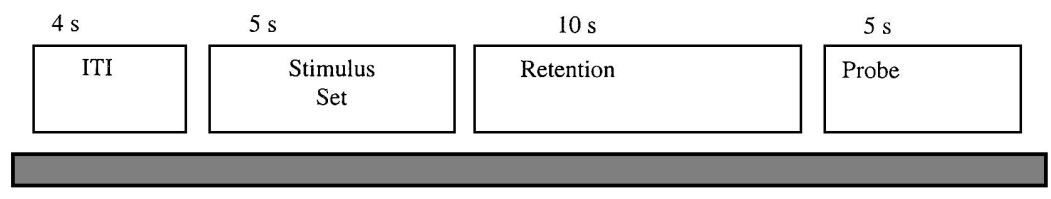

$\mathrm{B}$

\begin{tabular}{|c|c|c|c|c|}
\hline $4 \mathrm{~s}$ & $5 s$ & \multicolumn{2}{|c|}{$10 \mathrm{~s}$} & $5 \mathrm{~s}$ \\
\hline ITI & $\begin{array}{c}\text { Stimulus } \\
\text { Set }\end{array}$ & \multicolumn{2}{|c|}{ Retention } & Probe \\
\hline ITI & $\begin{array}{l}\text { Digit Span: } \\
\text { Stimulus } \\
\text { Presentation }\end{array}$ & $\begin{array}{l}\text { Digit Span: } \\
\text { Recall }\end{array}$ & $\begin{array}{l}\text { Digit Span: } \\
\text { Stimulus } \\
\text { Presentation }\end{array}$ & $\begin{array}{l}\text { Digit Span: } \\
\text { Recall }\end{array}$ \\
\hline
\end{tabular}

Figure 1. A: Visual task alone. B: Dual task with complete interference. ITI $=$ intertrial interval. 
single- and dual-task conditions (Snodgrass \& Corwin, 1988). The discrimination index, designated as DL, provided information regarding the individual's ability to discriminate between true positive and true negative probes. Higher DL values indicate better discrimination.

Repeated measures analyses of variance (ANOVAs), with age as the between-participants factor and task (single vs. dual) as the two-level within-participant variable, were carried out to determine whether agerelated differences in $\log _{e}$ reaction time and discrimination were increased in the dual-task compared with the single-task condition. Independentsamples $t$ tests were used as post hoc tests to examine whether age differences in $\log _{e}$ reaction time and discrimination were significant on the DVRT single- and dual-task conditions.

Factor analysis of the neuropsychological tests. For data reduction purposes, neuropsychological raw test scores of the young and older participants were submitted to principal-components factor analysis. A multivariate analysis of variance (MANOVA) was carried out on the neuropsychological factor scores and then followed by one-way ANOVAs to ensure that, as intended, individual differences on each factor would be defined in relation to age. Because the principal-components analysis was run on the correlation matrix, the raw scores were normalized on the basis of the distribution of the entire sample. Varimax rotation was used to derive orthogonal factor scores, and the minimum eigenvalue for extraction was set at 1 .

Prediction of age differences on the single-and dual-task conditions of the DVRT. To examine whether aging had an effect on dual-task performance independent of neuropsychological test performance, we orthogonalized age with respect to the neuropsychological factor scores. This was done by regressing age on the factor scores and using the residual as the new orthogonal age variable. The regression was statistically significant $(R=863), F(4,26)=18.981, p<.001$.

Multiple regression analyses examined whether individual and age differences on the neuropsychological factors predicted age-related performance variance on the DVRT. The neuropsychological factor scores served as the predictors that were entered simultaneously. $\log _{e}$ reaction time and discrimination indices of the DVRT in the single- and dual-task conditions served as the criteria in four different regression models. The orthogonal age variable was subsequently entered as an additional predictor into the regression models to determine whether aging had an effect on DVRT performance independent of the neuropsychological factor scores. The magnitude of the standardized beta coefficients was used to evaluate the relative contribution of each predictor to the variance of the dependent measures.

For all analyses in this article, the level of statistical significance was set at $\alpha=.05$. Tests for sphericity were carried out in all ANOVAs. HuynFeldt corrected significance levels are reported for any effect for which the sphericity test was significant. The degrees of freedom and statistical significance values reported for the independent-samples $t$ tests were corrected for unequal variance when necessary.

\section{Results}

\section{DVRT $\log _{e}$ Reaction Time and Discrimination Data}

Mean $\log _{e}$ reaction time (and the standard error of the mean), per age group, in the DVRT single- and dual-task conditions is presented in Figure 2.

A repeated measures ANOVA, with age as the between-participants factor and task as the within-participant variable, revealed significant main effects for age, $F(1,30)=80.346, p<.001$, and for task, $F(1,30)=6.943, p<.05$, and a significant Age $\times$ Task interaction, $F(1,30)=5.769, p<.05$, indicating that age differences increased from the single- to the dual-task condition of the DVRT. Independent-samples $t$ tests indicated statistically significant mean age differences in $\log _{e}$ reaction time on both the DVRT

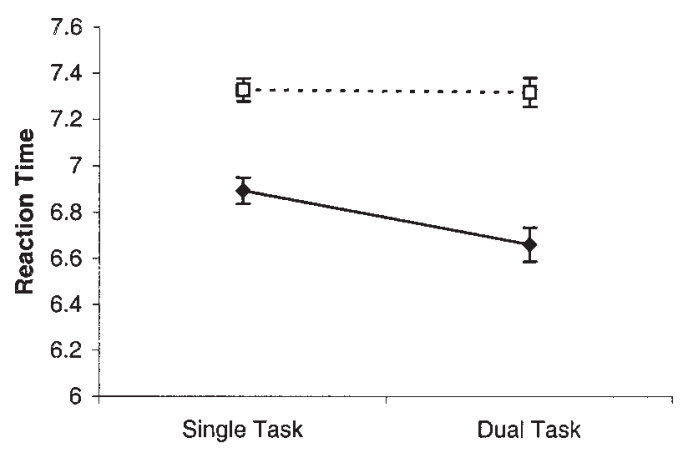

Figure 2. Mean $\log _{e}$ reaction time, per age group, in the single- and dual-task conditions of the delayed visual recognition task. Solid diamonds $=$ young participants; open squares $=$ older participants. Error bars represent standard error of the mean.

single-task condition, $t(27.977)=-6.615, p<.001$, and the dual-task condition, $t(28.611)=-7.651, p<.001$.

Mean discrimination (and the standard error of the mean), per age group, in the single- and dual-task conditions of the DVRT is presented in Figure 3.

A repeated measures ANOVA, with age as the between-participants factor and task as the within-participant variable, revealed significant main effects for age, $F(1,30)=55.528, p<.001$, and for task, $F(1,30)=56.783, p<.001$, and a significant Age $\times$ Task interaction, $F(1,30)=4.486, p<.05$, indicating that age differences in discrimination were increased in the dual-task condition. Independent-samples $t$ tests indicated statistically significant mean differences in discrimination between the young and older participants for both the DVRT single-task condition, $t(26.050)=-4.793, p<.001$, and the dual-task condition, $t(29.373)=6.983, p<.001$.

\section{Factor Analysis: Neuropsychological Tests}

As expected, group differences on the neuropsychological tests (raw scores) were statistically significant (see Table 1). We submitted the neuropsychological test scores of the young and older participants to principal-components factor analysis, yielding four orthogonal factors that accounted for a total of $73 \%$ of the variance of the neuropsychological tests (see Table 2).

The loading coefficients of the neuropsychological tests on the four factors were consistent with their purported use in the literature. ${ }^{1}$ The Attention/Executive factor consisted of the Digit Span Forward and Backward conditions, Trails Form A, Trails Forms B - A, and the Rey Complex Figure (Rey, 1941), copy version. On the Memory factor, we noted the highest loading coefficients for the SRT (Bushke \& Fuld, 1974) total recall and saving scores, the Rey 30-min delay, and DRS (Mattis, 1988) total score. The Motor Speed factor consisted primarily of the reaction time test (mean and standard deviation) and, to a lesser degree, of the Rey Complex Figure, copy version.

\footnotetext{
${ }^{1}$ The Boston Naming Test was not entered into the factor analysis because the young group was at ceiling on this test. Trails Form B was not entered into the factor analysis because it was redundant with the B - A index, which provided a more pure assessment of set shifting.
} 


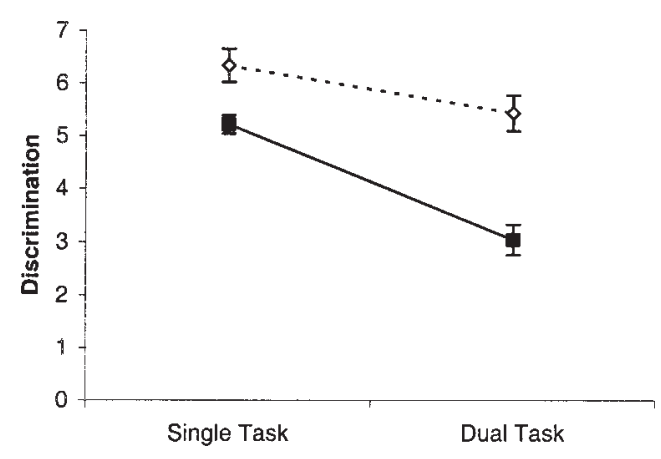

Figure 3. Mean discrimination $\left(d^{\prime}\right)$, per age group, in the single- and dual-task conditions of the delayed visual recognition task. Solid squares $=$ young participants; open diamonds $=$ older participants. Error bars represent standard error of the mean.

The NART (Nelson, 1982) had the largest loading coefficient on the Cognitive Status factor. We noted moderate loading coefficients on this last factor for the DRS (Mattis, 1988) total score and Trails B - A.

A MANOVA, with age as the between-participants factor and the four neuropsychological factors as the dependent measures, was statistically significant (Wilks's $\Lambda=.269), F(4,26)=17.707$, $p<.01$. Descriptive statistics (mean and standard error) and summary of the ANOVA results carried out on each neuropsychological factor are presented in Table 3.

There were significant age differences on the Attention/Executive and Memory factors, indicating that individual differences on these two factors were related to age. The differences between the young and older participants on the Motor Speed and Cognitive Status factors were not statistically significant, indicating that individual differences on these factors were not related to age.

\section{Multiple Regression Analyses}

The four neuropsychological factors and orthogonal age variable were used as predictors in four different regression models,

Table 2

Results of the Principal-Components Factor Analysis

\begin{tabular}{lrrrr}
\hline \multicolumn{1}{c}{ Variable } & $\begin{array}{c}\text { Attention/ } \\
\text { Executive }\end{array}$ & Memory & $\begin{array}{r}\text { Motor } \\
\text { Speed }\end{array}$ & $\begin{array}{c}\text { Cognitive } \\
\text { Status }\end{array}$ \\
\hline \% of variance & 22.608 & 20.776 & 18.169 & 11.636 \\
Tests & & & & \\
$\quad$ Digit Span (FW) & $\mathbf{. 7 9 5}$ & .066 & -.197 & .069 \\
Digit Span (BW) & $\mathbf{. 7 8 3}$ & .226 & -.070 & .066 \\
Trails (Form A) & $-\mathbf{. 7 3 1}$ & -.350 & .201 & .319 \\
Trails (B - A) &.- .536 & -.347 & .227 & .462 \\
Rey copy & $\mathbf{. 5 2 0}$ & .004 & -.460 & .210 \\
SRT saving score & -.004 & $\mathbf{. 8 9 4}$ & -.103 & .147 \\
SRT total score & .284 & $\mathbf{. 7 5 2}$ & -.128 & -.185 \\
Rey 30-min delay & .454 & $\mathbf{. 6 7 6}$ & -.037 & -.064 \\
DRS total score & .136 & $\mathbf{. 5 6 6}$ & -.163 & .466 \\
Reaction time $(S D)$ & -.151 & -.097 & $\mathbf{. 9 6 0}$ & .022 \\
Reaction time $(M)$ & -.217 & -.203 & $\mathbf{. 9 2 5}$ & .081 \\
NART total score & .024 & .049 & .025 & $\mathbf{. 8 6 2}$ \\
\hline
\end{tabular}

Note. Boldface indicates the highest loading coefficients per factor, and italic indicates coefficients above $.45 . \mathrm{FW}=$ Forward; BW $=$ Backward; Trails $=$ Trail Making Test; SRT $=$ Selective Reminding Test; DRS = Dementia Rating Scale; NART $=$ National Adult Reading Test.
Table 3

Mean and Standard Error Factor Scores per Age Group and Their Respective ANOVA Tests

\begin{tabular}{|c|c|c|c|c|c|c|}
\hline \multirow[b]{2}{*}{ Factor } & \multicolumn{2}{|c|}{ Young } & \multicolumn{2}{|c|}{ Older } & \multirow[b]{2}{*}{$M^{2}$} & \multirow[b]{2}{*}{$F(1,30)$} \\
\hline & $M$ & $S E$ & $M$ & $S E$ & & \\
\hline Attention/Executive & .568 & .18 & -.606 & .22 & 10.705 & $16.090 * *$ \\
\hline Memory & .525 & .11 & -.560 & .29 & 9.129 & $12.685 * *$ \\
\hline Motor Speed & -.221 & .07 & .236 & .35 & 1.654 & 0.209 \\
\hline Cognitive Status & -.121 & .16 & .129 & .33 & 0.482 & 0.493 \\
\hline
\end{tabular}

Note. $\quad \mathrm{ANOVA}=$ analysis of variance.

$* * p<.01$.

with $\log _{e}$ reaction time and discrimination indices in the DVRT single- and dual-task conditions serving as the dependent measures. Summary of the results of these regression models is presented in Table 4.

The neuropsychological factors were significant predictors of $\log _{e}$ reaction time and discrimination in the single- and dual-task conditions of the DVRT. The explained variance ranged from $57 \%$ to $73 \%$. With the exception of the regression model predicting discrimination in the single-task condition of the DVRT, the addition of the orthogonal age variable as a predictor was statistically significant, adding from $5 \%$ to $7 \%$ to the explained variance.

The regression coefficients of the neuropsychological factors and orthogonal age predicting $\log _{e}$ reaction time in the single- and dual-task conditions of the DVRT are presented in Table 5.

Table 5 reveals that the Attention/Executive and Memory factors as well as orthogonal age were significant predictors of reaction time in the single-task condition of the DVRT. In the dual-task condition, the four neuropsychological factors and orthogonal age were all significant predictors of $\log _{e}$ reaction time. This indicates that the neuropsychological factors were differentially related to performance in the single- and dual-task conditions of the DVRT. Examination of the standardized beta coefficients provided information with respect to the magnitude of the relation between the predictors and dependent measure. As judged by the coefficient size, the Memory factor had the largest contribution to predicting single-task performance, but the Attention/Executive factor emerged as the strongest predictor of dual-task performance.

The regression coefficients of the neuropsychological factors and orthogonal age predicting discrimination in the single- and dual-task conditions of the DVRT are presented in Table 6 .

Table 6 reveals that the four neuropsychological factors were significant predictors of discrimination in the single-task condition of the DVRT, whereas the orthogonal age was not. In the regression predicting discrimination in the dual-task condition, the Cognitive Status factor was no longer significant, but orthogonal age became a significant predictor.

Examination of the standardized beta coefficients revealed that Motor Speed was the strongest predictor of single-task performance. The relation of the Memory and Attention/Executive factors to discrimination varied as a function of task condition. Memory had a larger contribution to predicting single-task performance, but the Attention/Executive factor was the strongest predictor of dual-task performance. 
Table 4

Summary of Regression Analyses Predicting $\log _{e}$ Reaction Time and Discrimination in the DVRT Single- and Dual-Task Conditions Using the Four Neuropsychological Factors and Orthogonal Age

\begin{tabular}{|c|c|c|c|}
\hline Condition & $R^{2}$ & $\Delta R^{2}$ & $\Delta F$ \\
\hline \multicolumn{4}{|l|}{ Single task: $\log _{e}$ reaction time } \\
\hline Four neuropsychological factors & .570 & .570 & $8.627 * *$ \\
\hline Four neuropsychological factors and orthogonal age & .648 & .078 & $5.529 *$ \\
\hline \multicolumn{4}{|l|}{ Dual task: $\log _{e}$ reaction time } \\
\hline Four neuropsychological factors & .728 & .728 & $17.411 * *$ \\
\hline Four neuropsychological factors and orthogonal age & .779 & .051 & $5.741 *$ \\
\hline \multicolumn{4}{|l|}{ Single task: Discrimination } \\
\hline Four neuropsychological factors & .704 & .704 & $15.473 * *$ \\
\hline Four neuropsychological factors and orthogonal age & .706 & .002 & 0.152 \\
\hline \multicolumn{4}{|l|}{ Dual task: Discrimination } \\
\hline Four neuropsychological factors & .619 & .619 & $10.577 * *$ \\
\hline Four neuropsychological factors and orthogonal age & .690 & .071 & $5.688 *$ \\
\hline
\end{tabular}

Note. $\log _{e}=$ natural log transformation; DVRT $=$ delayed visual recognition task.

$* p<.05$. $* * p<.01$.

\section{Discussion}

The negative effect of old age on dual-task performance has been reported in previous research, but reasons for this finding remain controversial. The present study took a different approach to address this issue. Neuropsychological tests were used as a proxy for aging to examine whether performance on such tests can shed light on our understanding of why dual-task performance decreases as a function of old age.

The methodology and findings of the present study provide important contributions to the study of cognitive aging. This study is the first, to our knowledge, to test specific hypotheses with respect to the relation of individual differences on neuropsychological factors to age-related decline in chronometric and discrimination dual-task performance indices. We found a significant and informative relation between individual differences on empirically derived neuropsychological factors and dual-task performance in young and older individuals. Furthermore, the role of those factors in predicting performance varied as a function of the underlying constructs they presumably represented. Below we discuss our findings in relation to the three hypotheses the present study was designed to evaluate.

Table 5

Regression Coefficients of the Neuropsychological Factors and Orthogonal Age Predicting $\log _{e}$ Reaction Time in the DVRT Single-Task $(S-T)$ and Dual-Task $(D-T)$ Conditions

\begin{tabular}{lccrrr}
\hline \multirow{2}{*}{ Factor } & \multicolumn{2}{c}{$\beta$} & & \multicolumn{2}{c}{$B$} \\
\cline { 2 - 3 } \cline { 5 - 6 } \cline { 5 - 6 } \cline { 5 - 6 } & S-T & D-T & & S-T & D-T \\
\hline Attention/Executive $^{\mathrm{a}}$ & $-.474^{* *}$ & $-.579^{* * *}$ & & -0.552 & -0.960 \\
Memory $^{\mathrm{a}}$ & $-.511^{* *}$ & $-.470^{* *}$ & & -0.596 & -0.779 \\
Motor Speed $_{\text {Cognitive Status }}$ & .201 & $.236^{*}$ & & 0.234 & 0.392 \\
Orthogonal Age & .211 & $.340^{* *}$ & & 0.246 & 0.563 \\
\hline
\end{tabular}

Note. $\quad \log _{e}=$ natural $\log$ transformation; DVRT $=$ delayed visual recognition task.

${ }^{\mathrm{a}}$ Factors were significantly different by age.

$* p<.05 . \quad * * p<.01$.
Our first goal was to assess whether individual differences on neuropsychological tests were related to dual-task performance of young and older individuals. Regression analyses, predicting both reaction time and discrimination, revealed that $62 \%-73 \%$ of the variance in dual-task performance was accounted for by the neuropsychological factors. These findings confirmed the hypothesis that neuropsychological tests can serve as an adequate proxy for aging when evaluating the effect old age has on dual-task performance.

The second hypothesis examined whether the relation of the neuropsychological factors to performance on the DVRT varied as a function of task condition and the underlying constructs those factors presumably represented. Specifically, because the dual-task paradigm was designed to be consistent with Baddeley and Hitch's (1974) conceptualization of the central executive, we predicted that the role of the Attention factor would be more potent in the dual-task than in the single-task condition of the DVRT.

The DVRT (single-task condition) was modeled after the Sternberg paradigm (Sternberg, 1966, 1969), which is considered a measure of working memory. In light of the large body of literature reporting that working memory declines in the aging process (for a meta-analysis, see Verhaeghen, Marcoen, \& Goosens, 1993), one

Table 6

Regression Coefficients of the Neuropsychological Factors and Orthogonal Age Predicting Discrimination in the DVRT SingleTask $(S-T)$ and Dual-Task (D-T) Conditions

\begin{tabular}{lccrrr}
\hline \multirow{2}{*}{ Factor } & \multicolumn{2}{c}{$\beta$} & & \multicolumn{2}{c}{$B$} \\
\cline { 2 - 3 } \cline { 5 - 6 } \multicolumn{1}{c}{ S-T } & D-T & & S-T & \multicolumn{1}{c}{ D-T } \\
\hline Attention/Executive $^{\mathrm{a}}$ & $.374^{* *}$ & $.564^{* *}$ & & 0.556 & 1.046 \\
Memory $^{\mathrm{a}}$ & $.464^{* *}$ & $.393^{* *}$ & & 0.690 & 0.729 \\
Motor Speed & $-.536^{* *}$ & $-.322^{* *}$ & & -0.790 & -0.597 \\
Cognitive Status & $-.248^{*}$ & -.206 & & -0.369 & -0.382 \\
Orthogonal Age & -.042 & $-.266^{*}$ & & -0.004 & -0.035 \\
\hline
\end{tabular}

Note. $\quad$ DVRT $=$ delayed visual recognition task.

${ }^{\mathrm{a}}$ Factors were significantly different by age.

$* p<.05 . \quad * * p<.01$. 
could have expected the Memory factor to be the strongest predictor of age-related performance variability on the DVRT. This relation was observed, but only when predicting reaction time in the single-task condition of the DVRT (see Table 5). Motor Speed is a "built in" component of the DVRT, and general slowing (Salthouse, 1985, 1996) has been proposed as an underlying mechanism of age-related decline in cognitive test performance. Hence, one might have expected Motor Speed to be the most important predictor of age-related performance differences on the DVRT. This relation between Motor Speed and DVRT performance was found, but only when predicting discrimination in the single-task condition of the DVRT (see Table 6).

It was the Attention/Executive factor that consistently emerged as the most potent predictor of dual-task performance for both the $\log _{e}$ reaction time and discrimination indices. This finding has important theoretical implications. The presumed role of the central executive in dual-task processing is to allocate attentional resources to competing tasks (Baddeley, 1996; Baddeley et al., 2001; Baddeley \& Hitch, 1974). Consequently, from this theoretical perspective, it would be expected that the Attention factor plays an important role in predicting dual-task performance. The findings of the present study support this notion (but see Salthouse et al., 2003, regarding methodological issues relevant to the assessment of the executive functions). It may be further inferred that compromised ability in old age to meet increasing demands on attention in dual tasks results in disproportionate costs relative to age differences on the single tasks. Although the present study suggests that the Attention/Executive factor was a potent predictor of age-related differences in dual-task performance, it should be emphasized that this factor did not have an exclusive role in mediating such performance. The involvement of the other neuropsychological factors in predicting age-related differences in both task conditions attests to the complex cognitive demands inherent in the DVRT.

The measurable difference in recruiting the neuropsychological factors to predict single- and dual-task performance on the DVRT has important implications. First, it appears that although dual-task and working memory paradigms are often considered to be two related facets of executive function, their underlying demands are qualitatively different. In this study, we demonstrated that although the Memory and Motor Speed factors were the strongest predictors of performance on a working memory task (DVRT alone), the Attention factor was the most potent predictor of dual-task performance.

This finding is consistent with a recent study by Tun et al. (2002), in which neuropsychological tests with heavy attention and executive demands were related to recall accuracy when study conditions included a meaningful interference but not when the information to be remembered was studied in quiet optimal conditions. Hence, the association between dual-task performance and neuropsychological tests that rely on attention and set-shifting may be generalized beyond the task-specific requirements and experimental conditions that were unique to the present study.

The second and more general implication is concerned with the unfortunate dichotomy between cognitive sciences and neuropsychology. Whereas cognitive tasks have been traditionally designed to enhance knowledge about specific cognitive processes, neuropsychological tests have been used for clinical purposes and to characterize disease-related cognitive changes. The present study provides evidence of the utility of using these two approaches in concert to increase knowledge with respect to cognitive aging. Moreover, and in contrast to the dichotomy mentioned above, this investigation used individual differences in neuropsychological tests to explain a robust aging effect on a complex cognitive paradigm for which present accounts have provided discrepant and inconsistent views.

It is interesting to note parallelisms between the outcome of the ANOVA on the DVRT results and the outcome of the multiple regression models predicting those results. The DVRT had significant effects of age and task as well as their interaction. The regression models of single- and dual-task performance had predictors that differed by age that were significant in both models. This is consistent with the main effect of age in the ANOVA, to the extent that age-related neuropsychological variance predicts agerelated DVRT variance irrespective of task condition. Similarly, there were predictors that differed between the two tasks' models that are suggestive of the main effect of task, although these differed depending on which variable was being predicted. The order of the regression predictors in the models differed in priority, and the predictors involved did (Memory and Attention/Executive) and did not (Motor Speed) differ by age. These reordering effects are consistent with the interaction of age and task. The implication of these patterns is that the task and age effects on the DVRT itself may be mediated by individual differences in several cognitive domains.

The third hypothesis addressed the issue of whether aging has an effect on dual-task performance that is independent of the variance captured by the neuropsychological factors. With the exception of predicting discrimination in the single-task condition, the orthogonal age variable added $5 \%-7 \%$ to the explained variance in DVRT performance. This suggests that age had an effect on DVRT performance that was not accounted for by the four neuropsychological factors, at least as measured in the present study. However, the independent contribution of age to the prediction of DVRT performance was relatively small. Although it is possible that a more comprehensive neuropsychological battery than the one used in the present study might have served as a better proxy for age, other factors, such as endurance or arousal, that are not typically captured by cognitive measures may have been represented by the orthogonal age variable and its independent prediction of variability in DVRT performance.

In evaluating the contribution of neuropsychological tests to our understanding of age-related differences in dual-task performance, it was necessary to address methodological issues relevant to the assessment of dual-task performance in general and age-related costs in particular. Consistent with previous research (e.g., Baddeley, 2002; Hartley, 1992), the dual-task paradigm in this study comprised two single tasks that were presumably processed via two different perceptual modalities. The participants were well trained in both the single- and dual-task conditions of the DVRT. Furthermore, the older participants demonstrated high accuracy (93\% correct) when performing the DVRT in the single-task condition, indicating that they had achieved an adequate level of mastery before they were subjected to the demands of the dual task. Further support for the argument that group differences in the dual-task condition were not primarily accounted for by performance differences in the single-task condition came from the significant Group $\times$ Task interactions observed for both the $\log _{e}$ 
reaction time and discrimination data. Addressing the above methodological issues has led to some level of assurance that individual differences on neuropsychological factors have shed light on our understanding of the observed negative effect of aging on dualtask performance.

In conclusion, individual differences in neuropsychological tests predicted dual-task performance of young and older individuals. Although the neuropsychological factors served as adequate proxy for the effect of aging on dual-task costs, age had a small but additional independent effect on performance. The Attention/Executive factor had an important, although not exclusive, role in mediating dual-task performance. These findings may be viewed as consistent with conceptualization of the putative central executive as important in allocating attentional resources to competing task demands. The findings of the present study suggest that compromised function of the central executive might underlie the negative effect aging has on dual-task performance.

\section{References}

Anderson, N. D. (1999). The attentional demands of encoding and retrieval in young and older adults: 2 . Evidence from secondary task reaction time distributions. Psychology and Aging, 14, 645-655.

Anderson, N. D., Craik, F. I. M., \& Naveh-Benjamin, M. (1998). The attentional demands of encoding and retrieval in younger and older adults: 1. Evidence from divided attention costs. Psychology and Aging, 13, 405-423.

Baddeley, A. (1996). Exploring the central executive. Quarterly Journal of Experimental Psychology: Human Experimental Psychology, 49(A), $5-28$.

Baddeley, A. (2002). Is working memory still working? European Psychologist, 7, 85-97.

Baddeley, A. D., Baddeley, H. A., Bucks, R. S., \& Wilcock, G. K. (2001). Attentional control in Alzheimer's disease. Brain, 124, 1492-1508.

Baddeley, A. D., \& Hitch, G. (1974). Working memory. In G. H. Bower (Ed.), The psychology of learning and motivation (Vol. 8, pp. 47-89). New York: Academic Press.

Batsakes, P. J., \& Fisk, A. D. (2000). Age-related differences in dual-task visual search: Are performance gains retained? Journal of Gerontology: Psychological Sciences, 55B, 332-342.

Bull, R., \& Scerif, G. (2001). Executive functioning as a predictor of children's mathematics ability: Inhibition, switching, and working memory. Developmental Neuropsychology, 19, 273-293.

Bushke, H., \& Fuld, P. A. (1974). Evaluating storage, retention, and retrieval in disordered memory and learning. Neurology, 24, 1019-1025.

Cohen, J. D., MacWhinney, B., Flatt, M., \& Provost, J. (1993). PsyScope: An interactive graphic system for designing and controlling experiments in the psychology laboratory using Macintosh computers. Behavior Research Methods, Instruments, \& Computers, 25, 257-271.

Crossley, M., \& Hiscock, M. (1992). Age-related differences in concurrent task performance of normal adults: Evidence for a decline in processing resources. Psychology and Aging, 7, 499-506.

Doran, S. M., Van Dongen, H. P. A., \& Dinges, D. F. (2000). Sustained attention performance during sleep deprivation: Evidence of state instability. Archives of Italiennes de Biologie, 138, 1-15.

Gick, M. L., Craik, F. I. M., \& Morris, R. G. (1988). Task complexity and age differences in working memory. Memory \& Cognition, 16, 353-361.

Glass, J. M., Shumacher, E. H., Lauber, E. J., Zurbriggen, E. L., Gmeindl, L., Kieras, D. E., \& Meyer, D. E. (2000). Aging and the psychological refractory period: Task-coordination strategies in young and old adults. Psychology and Aging, 15, 571-595.

Hartley, A. A. (1992). Attention. In F. I. M. Craik \& T. A. Salthouse (Eds.), Handbook of aging and cognition (pp. 1-49). Hillsdale, NJ: Erlbaum.
Hartley, A. A. (2001). Age differences in dual-task interference are localized to response-generation processes. Psychology and Aging, 16, 4754.

Hartley, A. A., \& Little, D. M. (1999). Age-related differences and similarities in dual-task interference. Journal of Experimental Psychology: General, 128, 416-449.

Holtzer, R., Stern, Y., \& Rakitin, C. B. (in press). Age-related differences in executive control of working memory. Memory \& Cognition.

Kortling, J. E. (1991). Effects of skill integration and perceptual competition on age-related differences in dual-task performance. Human Factors, 33, 35-44.

Kramer, A. F., Hahn, S., \& Gopher, D. (1999). Task coordination and aging: Explorations of executive control processes in the task switching paradigm. Acta Psychologica, 101, 339-378.

Lezak, M. D. (1995). Neuropsychological assessment (3rd ed.). New York: Oxford University Press.

Li, K. Z. H., Lindenberger, U., Freund, A. M., \& Baltes, P. B. (2001). Walking while memorizing: Age-related differences in compensatory behavior. Psychological Science, 12, 230-237.

Malapani, C., Pillon, B., Dubois, B., \& Agid, Y. (1994). Impaired simultaneous cognitive task performance in Parkinson's disease: A dopaminerelated dysfunction. Neurology, 44, 319-326.

Mattis, S. (1988). Dementia Rating Scale (DRS). Odessa, FL: Psychological Assessment Resources.

McDowell, S., Whyte, J., \& D'Esposito, M. (1997). Working memory impairments in traumatic brain injury: Evidence from a dual-task paradigm. Neuropsychologia, 35, 1341-1353.

McDwod, J. M., \& Craik, F. I. M. (1988). Effects of aging and task difficulty on divided attention performance. Journal of Experimental Psychology: Human Perception and Performance, 14, 267-280.

McDwod, J. M., \& Shaw, R. J. (2000). Attention. In F. I. M. Craik \& T. A. Salthouse (Eds.), Handbook of aging and cognition (2nd ed., pp. 221292). Hillsdale, NJ: Erlbaum.

McDwod, J. M., Vercruyssen, M., \& Birren, J. E. (1991). Aging, divided attention and dual-task performance. In D. L. Damos (Ed.), Multiple task performance (pp. 387-414). Bristol, PA: Taylor \& Francis.

Meyer, D. E., \& Kieras, D. E. (1997). A computational theory of executive cognitive processes and multiple task performance: Part 2. Accounts of psychological refractory-period phenomena. Psychological Review, 104, 749-791.

Miyake, A., Friedman, N. P., Emerson, M. J., Wizki, A. H., Howerter, A., \& Wager, T. D. (2000). The unity and diversity of executive functions and their contributions to complex "frontal lobe" tasks: A latent variable analysis. Cognitive Psychology, 41, 49-100.

Morris, R. G., Gick, M. L., \& Craik, F. I. M. (1988). Processing resources and age differences in working memory. Memory \& Cognition, 16, 362-366.

Navon, D., \& Miller, J. O. (1987). The role of outcome conflict in dual-task interference. Journal of Experimental Psychology: Human Perception and Performance, 13, 438-448.

Nelson, H. E. (1982). National Adult Reading Test (NART): Test manual. Windsor, England: NFER Nelson.

Norman, D. A., \& Shallice, T. (1980). Attention to action: Willed and automatic control of behavior (Center for Human Information Processing Rep. No. 99). San Diego: University of California.

Nyberg, L., Nilsson, L. G., Olofsson, U., \& Backman, L. (1997). Effects of division of attention during encoding and retrieval on age differences in episodic memory. Experimental Aging Research, 23, 137-143.

Rey, A. (1941). L'examen psychologique dans les cas d'encephalopathie traumatique [Psychological examination of traumatic encephalopathy]. Archives de Psychologie, 28, 286-340.

Salthouse, T. A. (1985). A theory of cognitive aging. Amsterdam: North Holland. 
Salthouse, T. A. (1996). The processing speed theory of adult age differences in cognition. Psychological Review, 103, 403-428.

Salthouse, T. A., Atkinson, T. M., \& Berish, D. E. (2003). Executive functioning as a potential mediator of age-related cognitive decline in normal aging. Journal of Experimental Psychology: General, 132, 566594.

Salthouse, T. A., Rogan, J. D., \& Prill, K. A. (1984). Division of attention: Age differences on a visually presented memory task. Memory \& Cognition, 12, 613-620.

Snodgrass, J. G., \& Corwin, J. (1988). Pragmatics of measuring recognition memory: Application to dementia and amnesia. Journal of Experimental Psychology: General, 117, 34-50.

Somberg, B. L., \& Salthouse, T. A. (1982). Divided attention abilities in young and old adults. Journal of Experimental Psychology: Human Perception and Performance, 8, 651-663.

Stern, Y., Andrews, H., Pittman, J., Sano, M., Tatemichi, T., Lantigua, R., \& Mayeux, R. (1992). Diagnosis of dementia in a heterogeneous population: Development of a neuropsychological paradigm-based diagnosis of dementia and quantified correction for the effects of education. Archives of Neurology, 49, 453-460.

Sternberg, S. (1966). High-speed scanning in human memory. Science, 153, 652-654.

Sternberg, S. (1969). The discovery of processing stages: Extensions of Donders' method. Acta Psychologica, 30, 276-315.

Stuss, D. T., \& Alexander, M. P. (2000). Executive functions and the frontal lobes: A conceptual view. Psychological Research, 63, 289-298.

Stuss, D. T., \& Levine, B. (2002). Adult clinical neuropsychology: Lessons from studies of the frontal lobes. Annual Review of Psychology, 53, 401-433.
Tun, P. A., O'Kane, G., \& Wingfield, A. (2002). Distraction by competing speech in young and older adult listeners. Psychology and Aging, 17, 453-467.

Tun, P. A., \& Wingfield, A. (1994). Speech recall under heavy load conditions: Age, predictability, and limits on dual-task interference. Aging, Neuropsychology, and Cognition, 1, 29-44.

Verhaeghen, P., \& Cerella, J. (2002). Aging, executive control, and attention: A review of meta-analyses. Neurosciences and Biobehavioral Reviews, 26, 849-857.

Verhaeghen, P., Marcoen, A., \& Goosens, L. (1993). Facts and fiction about memory aging: A quantitative integration of research findings. Journal of Gerontology: Psychological Sciences, 48, 157-171.

Verhaeghen, P., Steitz, D. W., Sliwinski, M. J., \& Cerella, J. (2003). Aging and dual-task performance: A meta-analysis. Psychology and Aging, 18, 443-460.

Wechsler, D. (1997). Wechsler Adult Intelligence Scale-Third Edition (WAIS-III). San Antonio, TX: Psychological Corporation.

Whiting, W. L., \& Smith, A. D. (1997). Differential age-related processing limitations in recall and recognition tasks. Psychology and Aging, 12, 216-224.

Wickens, C. D., Braune, R., \& Stokes, A. (1987). Age differences in the speed and capacity of information processing: 1. A dual-task approach. Psychology and Aging, 2, 70-78.

Received July 25, 2003

Revision received January 6, 2004

Accepted January 8, 2004

\section{E-Mail Notification of Your Latest Issue Online!}

Would you like to know when the next issue of your favorite APA journal will be available online? This service is now available to you. Sign up at http://watson.apa.org/ notify/ and you will be notified by e-mail when issues of interest to you become available! 